\title{
PROBLEMS IN INDOOR MAPPING AND MODELLING
}

\author{
Sisi Zlatanova ${ }^{\mathrm{a}}$, George Sithole ${ }^{\mathrm{b}}$, Masafumi Nakagawa ${ }^{\mathrm{c}}$, Qing Zhu ${ }^{\mathrm{d}}$ \\ ${ }^{a}$ GISt, OTB, Delft University of Technology, Jaffalaan 9, 2628 BX Delft, The Netherlands, Email: s.zlatanova@tudelft.nl \\ ${ }^{\mathrm{b}}$ Geomatics Division, School of Architecture, Planning and Geomatics, University of Cape Town, Private Bag X3, Rondebosch, \\ 7701, South Africa, Email: george.sithole@uct.ac.za \\ ${ }^{c}$ Department of Civil Engineering, College of Engineering, Shibaura Institute of Technology, 3-7-5, Toyosu, Koto-ku, Tokyo, 135- \\ 8548, Email: mnaka@ shibaura-it.ac.jp \\ ${ }^{\mathrm{d}}$ Faculty of Geosciences and Environmental Engineering of Southwest Jiaotong University, 611756, Chengdu, Sichuan, P.R.China, \\ Email: zhuq66@263.net
}

Commission IV, WG IV/7

KEY WORDS: Indoor, Mapping, Modelling, Navigation, Visualisation, Application

\begin{abstract}
:
Research in support of indoor mapping and modelling (IMM) has been active for over thirty years. This research has come in the form of As-Built surveys, Data structuring, Visualisation techniques, Navigation models and so forth. Much of this research is founded on advancements in photogrammetry, computer vision and image analysis, computer graphics, robotics, laser scanning and many others.
\end{abstract}

While IMM used to be the privy of engineers, planners, consultants, contractors, and designers, this is no longer the case as commercial enterprises and individuals are also beginning to apply indoor models in their business process and applications. There are three main reasons for this. Firstly, the last two decades have seen greater use of spatial information by enterprises and the public. Secondly, IMM has been complimented by advancements in mobile computing and internet communications, making it easier than ever to access and interact with spatial information. Thirdly, indoor modelling has been advanced geometrically and semantically, opening doors for developing user-oriented, context-aware applications. This reshaping of the public's attitude and expectations with regards to spatial information has realised new applications and spurred demand for indoor models and the tools to use them.

This paper examines the present state of IMM and considers the research areas that deserve attention in the future. In particular the paper considers problems in IMM that are relevant to commercial enterprises and the general public, groups this paper expects will emerge as the greatest users IMM.

The subject of indoor modelling and mapping is discussed here in terms of Acquisitions and Sensors, Data Structures and Modelling, Visualisation, Applications, Legal Issues and Standards. Problems are discussed in terms of those that exist and those that are emerging. Existing problems are those that are currently being researched. Emerging problems are those problems or demands that are expected to arise because of social changes, technological advancements, or commercial interests.

The motivation of this work is to define a set of research problems that are either being investigated or should be investigated. These will hopefully provide a framework for assessing progress and advances in indoor modelling. The framework will be developed in the form of a problem matrix, detailing existing and emerging problems, their solutions and present best practices. Once the framework is complete it will be published online so that the IMM community can discuss and modify it as necessary. When the framework has reached a steady state an empirical benchmark will be provided to test solutions to posed problems. A yearly evaluation of the problem matrix will follow, the results of which will be published.

\section{INTRODUCTION}

Research in support of indoor mapping and modelling (IMM) has been active for over thirty years. This research has come in the form of As-Built surveys, Data structuring, Visualisation techniques, Navigation models and so forth. Much of this research is founded on advancements in photogrammetry, computer vision and image analysis, computer graphics, robotics, laser scanning and many others.

While IMM used to be the privy of engineers, planners, consultants, contractors, and designers, this is no longer the case as commercial enterprises and individuals are also beginning to include indoor models in their business process and applications. There are two main reasons for this. Firstly, the last two decades have seen greater use of indoor spatial information by the public (Worboys 2011). Secondly, IMM has been complimented by advancements in mobile computing and internet communications, as well as the seamless indoor and outdoor positioning, making it easier than ever to access and interact with indoor spatial information. This reshaping of the public's attitude and expectations with regards to spatial information has realised new applications and spurred demand for indoor models and the tools to use them.

This paper examines the present state of IMM and considers the research areas that deserve attention in the future. In particular the paper considers problems in IMM that are relevant to the public, a group this paper expects will emerge as the greatest users IMM. The motivation of this work is to define a set of research problems that are either being investigated or should be investigated. 
These will hopefully provide a framework for assessing progress and advances in indoor modelling. The framework will be developed in the form of a problem matrix, detailing existing and emerging problems, their solutions and present best practices. Once the framework is complete it will be published online so that the IMM community can discuss and modify it as necessary. When the framework has reached a steady state an empirical benchmark will be provided to test solutions to posed problems. A yearly evaluation of the problem matrix will follow, the results of which will be published.

\section{THE STATE OF IMM, EXISTING AND EMERGING PROBLEMS}

The subject of indoor modelling and mapping is discussed here in terms of Acquisitions and Sensors, Data Structures and Modelling, Visualisation, Navigation, Applications, Legal Issues and Standards.

Problems will be discussed in terms of those that exist and those that are emerging. Existing problems are those that are currently being researched. Emerging problems are those problems or demands that are expected to arise because of social changes and technological advancements (see Fig 1).

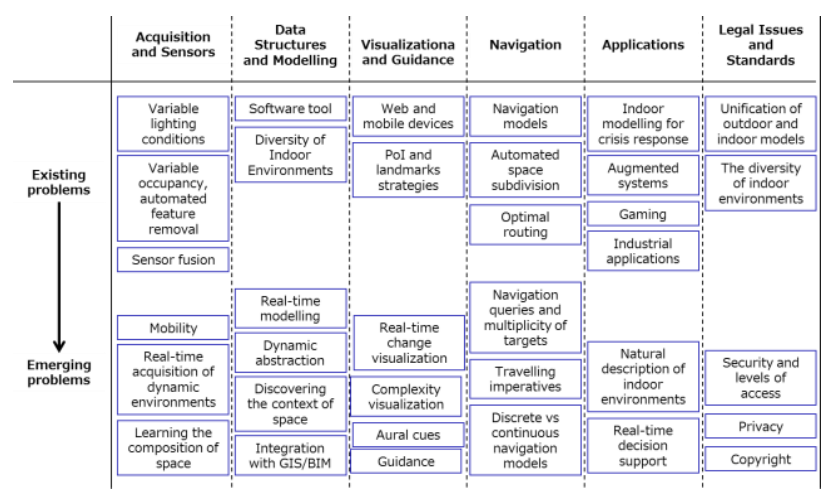

Figure 1: Existing problems

\subsection{Acquisition and Sensors}

Acquisition here refers to the measurement techniques, sensors, media, and platforms use to acquire raw data describing the geometry and radiometry of indoor environments.

The methods of acquisition of indoor environments have largely been adapted from acquisition methods of outdoor environments. Naturally because of space constraints, integrated systems of sensors are placed on robots, trolleys, or backpacks. These have been successful. The greatest challenge has been the localisation and registration of acquired data (Kolodziej and Hjelm, 2006). These challenges have been overcome by Simultaneous Localisation and Mapping (SLAM), the use of Inertial Measurement Units, sonar sensors (Girard et al 2011), and wireless positioning systems to name a few.

\section{Existing problems}

Problem A1, Variable lighting conditions:

While active measurement systems like lidar are finding greater used in indoor measurement systems, the use of image/vision based acquisition systems will continue because of their higher resolution. A major problem in vision based systems is feature measurement and detection under variable lighting conditions, which is a characteristic of indoor environments.

Problem A2, Variable occupancy, automated feature removal: Many measurement systems are designed for environments that contain as few visual obstructions as possible. Indoor environments are often busy and cluttered. Measurement under these conditions is difficult, particularly for vision based systems. Under these conditions a measurement system has to be able to achieve two goals, (1) detect and remove obstacles automatically and (2) measure the features of interest (e.g., walls).

Problem A3, Sensor fusion:

It's known that acquisition systems that are able to combine the strengths of different sensor systems are able to deliver more reliable results. The development/improvement of new sensors and the combination of these sensors is an on-going research.

\section{Emerging problems}

Problem A4, Mobility:

Often lay users are considered to be only interested in the application of indoor models. However, in indoor environments the acquisition and application of indoor data is entwined because the user is immersed in the environment. An example of this are augmented systems. Therefore, as mobile devices gain in computing power, hardware and software tools will have to be developed to make it possible to acquire indoor spaces using mobile devices.

Problem A5, Real-time acquisition of dynamic environments: The modelling of dynamic environments, i.e. environments in which there are many moving objects (e.g., crowded rooms) or environments in which space reforms (e.g., industrial plants) is presently done off-line. The challenge is to do this in real-time and on mobile devices.

An extension of this problem is the detection and measurement of changes in space and the real-time updating of previously acquired models.

Problem A6, Learning the composition of space:

Generally, the focus of indoor mapping has been to acquire the structures that define space (Becker, et al. 2009). These structures include walls, windows, load bearing pillars and so forth. In an indoor environment the objects that compose space are also of interest. The detection of humans in imagery is an active research area. But this needs to be expanded to the furniture that composes living spaces. This will mostly likely require building content retrieval systems in indoor acquisition system.

\subsection{Data Structures and Modelling}

The raw data from the acquisition processes have to be edited, fused, reformed, formatted and attributed. Editing involves the removal of unwanted data or artefacts from the raw data. The various data are then registered or fused to deliver an amalgamated scene of an indoor environment. In the reforming step, features are extracted from this amalgamated scene. These features are often represented by spatial models that range from simple 2D drawings to parametric 3D object models. During formatting, topological information is extracted and stored in an explicit format. Finally semantic information is attached to the extracted features. The extracted features, together with the 
topological and semantic information form the basic indoor model. This production pipeline is well established.

\section{Existing Problems}

Problem B1, Software tools:

The software tools used for indoor modelling are largely generic CAD or computer graphics tools. There are very few dedicated tools for processing indoor data and where they exist they are usually found in research labs. For example what is presently absent or requires improvements are intelligent tools to fuse the various sources and forms indoor data, such as imagery, point clouds, vector layers, etc.,. Understandably a 'one size fits all' tool is problematic because there are a diverse number of indoor environments (e.g., rooms, tunnels, factories, pipes, etc.). Moreover, any particular environment will differ depending on lighting conditions and occupancy.

Problem B2, Diversity of Indoor Environments:

Overcoming the above problem will first require a study of the different types of indoor environments with the objective of cataloguing and categorising them. Once such taxonomy exists, coherent tools can be designed around the catalogue.

\section{Emerging Problems}

Problem B3, Real-time modelling:

This problem is an expansion on problem A5. The automatic acquisition of dynamic environments has to be supported by suitable spatial data models.

Problem B4, Dynamic abstraction:

The emphasis of tradition data acquisition is to capture as much details as possible, which results in a lot of data. Nevertheless, for the average users in IMM, this often offers more information than needed so that will cost excessive computation efforts and even leads to erroneous results. Therefore, techniques will have to be developed that are able to offer users models with the proper amount of details that they can manage.

Problem B5, Discovering the context of space:

"Context varies according to application constraints, taking into account the way users act in the environment, as well as the interfaces to interact with." (Afyouni et al, 2012). This problem is allied to problem A6. Because of their application and composition, indoor environments will differ. Before automated acquisition and modelling can begin this difference has to be accounted for and operator. Typically the operator will choose appropriate hardware and software parameters. An example for this might be accounting for differences in lighting conditions or identifying the context of an environment based on the objects contained in the environment. The challenge is to discover the context of environments automatically so that the devices used for acquisition and modelling become universal.

Problem B6, Integration with GIS/BIM:

The CityGML standard in GIS community and the IFC standard in BIM are popular in both industry and academic world, because they are composed of rich geometry and semantics representing a building from outdoor to indoor. Therefore, they are the solid foundation of the data model for IMM. However, the two standards differ in many aspects such as the means of geometric representation and taxonomies. As the available datasets are surging, they have to be supported and integrated for IMM applications. Moreover, the spaces that are essential for IMM are not well defined in these models and it is not trivial to extract this information fully automatically because of the inconsistences during modelling, which is also to be tackled.

For example, BIM was proposed in the field of building design as the international industry semantic standard (NBIMS-1.0, 2007), being capable of providing an "authoritative semantic definition of building elements, spatial relationships, and quantities and properties of building components". The insufficiencies of indoor navigation approaches caused by the existing GIS system can be overcome by making use of the advanced semantic and geometric information that are available in BIM. With the purpose of providing a successful 3D indoor navigation, it is necessary to transfer $3 \mathrm{D}$ geometric and semantic information from BIM into a 3D GIS environment (Isikdag, 2006). The challenge is to maintain the consistency of automatic change of LOD models

\subsection{Visualisation}

Visualisation refers to the depiction of indoor models. Computer graphics and visualisation are very well documented fields. With regards to IMM, the most important contributions are in the field of augmented reality with particular application to mobile devices.

\section{Existing problems}

Problem C1, Web and mobile devices:

Desktop systems have traditionally been the devices for visualising $3 \mathrm{D}$ indoor models. However, with the popularity of mobile devices new visualisation methods will have to be realised for devices with relatively small screen real estate.

Problem C2, PoI and landmarks strategies:

Various strategies of Points of Interest and landmarks have been investigated to facilitate outdoor navigation (Peters et al 2010). However these cannot be readily applied for indoor environments. Different indoor objects might be identified as landmarks or points of interest for the purpose of distinct applications. These objects might be even not that characteristic as the traditional outdoor landmarks.

\section{Emerging problems}

Problem C3, Real-time change visualisation:

This problem is allied to problems A5 and B3. Visualising change in $2 \mathrm{D}$ is fairly straight forward. Visualising change in $3 \mathrm{D}$ is a little more difficult. The challenge is how to visualise change in $3 \mathrm{D}$ in real-time. This is particularly important for work with mobile devices.

Problem C4, Complexity visualisation:

The amounts of information that are available to the user will certainly increase. The challenge is to manage the display of this information without reducing its complexity.

\section{Problem C5, Aural cues:}

The use of audio cues in 3D entertainment is not new. However, it hasn't been widely applied in geospatial applications. IMM is one application in which aural cues can offer many benefits. Although it is not visualisation, aural cues just like visualisation aim to serve to the user a perception of the environment.

Aural cues have one major advantage over visualisation, in that they can convey to the user that which is not in the users field of view. That is to say that with aural cues users can localise 
objects that they cannot see. In confined spaces this can be a big advantage. Moreover, aural cues (e.g., reverb) can be used to present to users the occupancy of space.

\subsection{Navigation}

Navigation relates to the tasks of path-finding and route planning within and indoor environment. Path-finding are those functions that are used to discover the routes between any two points; if they exist. Route planning is the optimization of routes between points given certain constraints.

\section{Existing Problems}

Problem D1, Navigation models:

Before navigational information can be extracted, the geometric model of the indoor environment has to be attributed/semantically enriched and structured in a geometric form ideal for navigational. This structuring mostly yields Network, Grid and Force Field based navigational models. These navigational models differ in their treatment of the continuity of indoor space and by extension the range of movements that can be modelled in this space.

Problem D2, Automated space subdivision:

Network and Grid based form the basis of most indoor navigation models. These models require that the geometry of indoor space be subdivided in an automated fashion. Devising heuristics for space subdivision is non-trivial as subdivisions are application specific and no one subdivision is better than another.

\section{Problem D3, Optimal routing:}

In outdoor environments, users will often desire the shortest distance between their location and their destination. However, depending on the mode of transport, the condition of the routes and other factors a user may opt for alternative routes. The same applies to indoor environments. However, indoor environments differ in that the travel distances are shorter and hence shortest paths are not as critical. Instead factors such as the risk of getting lost, route avoidance and so forth become more important. Devising algorithms that can provide user 'friendly' routes is an area of investigation.

\section{Emerging Problems}

Problem D4, Navigation queries and multiplicity of targets: Navigation queries often seek to answer the question, "What is the best route to travel from a point $\mathbf{A}$ to a point $\mathbf{B}$ ?" Here the best route is determined by the cost of the route in terms of distance, accessibility, mode of transport, etc. In indoor environments these queries are constrictive because in these environments the intent to travel is seldom as simple as moving from a point $\mathbf{A}$ to a point $\mathbf{B}$. In many cases several users must be navigated simultaneously, or the target points are several or the target points are dynamic. Therefore, indoor navigation queries have to be expanded to accommodate a range of more complex/dynamic scenarios such as a case of fire emergency.

Problem D5, Travelling imperatives:

Most of the current navigation models operate on the presumption that users are either compelled or impelled to move from one point to another within an indoor space. These models exclude the intentions of the user in the routing analysis. As IMM becomes more main stream users will appreciated to have the opportunity to customize their routes to better fit their needs, and this will require navigational models designed around user intent.

Problem D6, Discrete vs continuous navigation models: Whether network or grid based, many of the current navigation models are discrete. The user may have the illusion that they can navigate to any point in the model, but in fact they can't. Whether this is a problem remains to be seen. But as 3D indoor models become more detailed and interactive it will become known whether continuous navigation models are required.

Problem D7, Guidance:

Many strategies exit to specifying the path to be followed: visualisation on a $2 \mathrm{D}$ map or within a $3 \mathrm{D}$ view, augmented reality, textual instructions, voice or video. As the guidance is the last step in the navigation process and intended to help the user, it should be most adapted to context of the user: gender, interests, preferences, tasks to be completed, environmental conditions and so forth. The user-centred interfaces become increasingly important.

\subsection{Applications}

\section{Existing Problems}

Problem E1, Indoor modelling for crisis response:

Evacuating an area during a time of crisis has for decades been an active area of research. Applications for this purpose have to be able to determine safe routes and guide users through safe routes. This requires sensors to capture the state of the indoor environment, relate sensory information to the indoor model, determine viable escape routes and finally convey the escape route to the user, either visually or aurally.

Additional to this, 3D indoor models can be used crisis simulations.

Problem E2, Augmented systems:

Augmented systems superimpose CGI over real world imagery with the purpose of offering the viewer an enhanced or more informative image of the real world. As more information becomes available and new visualising technologies are developed, new methods of augmentation will also have to be considered.

\section{Problem E3, Gaming:}

Traditionally 3D games have relied on the design of virtual indoor environments. However, as 3D indoor models become common, it is likely that there will be greater use of 'real' indoor models. Eventually as augmented systems mature, it can be expected that a new genre of games will emerge in which games are played in real indoor environments with the benefit of augmented reality.

Problem E4, Industrial applications:

This problem relates to problem A6. Measuring and discovering the composition of space, i.e. determining the content of indoor space will allow greater interactivity with the space. This applies in particular to industrial and manufacturing environments where users have to operate/navigate machinery in confined spaces.

\section{Emerging Problems}

Problem E5, Natural description of indoor environments (semantics): 
This is an extension of problem B5. If the context of an environment can be discerned then it stands to reason that with further work we can describe the environment in natural language. This then allows space to be described to users in ways that are familiar to them.

Problem E6, Real-time decision support:

This problem builds on problem E5. A user interacts, navigates or uses an indoor environment with a purpose. If an indoor environment can be described in natural language, and provided that an $\mathrm{AI}$ is available, then a user's decisions in the indoor environment can be supported by the AI.

\subsection{Legal Issues and Standards}

As indoor models are increasingly applied to consumer applications, various legal and security issues arise. In addition standards have to be developed to facilitate the use of the indoor models in a variety of applications (Lee et al, 2013).

\section{Existing Problems}

Problem F1, Unification of outdoor and indoor models: The development of modelling standards for 3D city models is well developed, largely because outdoor data acquisition and modelling has been active for many years. Indoor modelling is a more recent activity and the models are not as matured as those of outdoor models. Ultimately a seamless transition between outdoor and indoor models is desired, and this is an active area of research.

Problem F2, The diversity of indoor environments:

As already mentioned indoor environments are diverse. This diversity may have led to a tendency to model indoor environments differently. There may therefore be a need to standardise modelling across the diverse indoor environments.

\section{Emerging Problems}

Problem F3, Security and levels of access:

One of the main concerns raised has about indoor models over the web and on mobile devices has been that of security. This can be overcome by providing users permissions to parts of an indoor model; however the protocols and standards for this have to be developed.

\section{Problem F4, Privacy:}

In this paper the mapping of indoor environments using mobile devices has been mentioned several times. Indoor environments are often occupied and this raises the issue of privacy. The issue of privacy is very important and the challenge is to develop and apply technology in ways that protect privacy. Real-time acquisition and modelling may provide a solution here in that the source data can be immediately destroyed after the model is created, leaving behind a model free of private information.

Problem F5, Copyright:

As repositories of user imagery (of indoor environments) grows this will allow indoor environments to be recreated. Therefore, it is expected that at some point in the future open source indoor models, premised on open maps concepts such as Open Street Maps, will start to appear. This will then give rise to questions of ownership of the models created from crowd sourced imagery.

\section{DISCUSSION}

The problems discussed above are only a means to an end. Ultimately the goal of IMM is to allow users to interact with indoor environments in ways that enhance their use of space.

Many of the problems are interlinked, i.e. and enhancement in one problem will provide improvements in the other. This is particularly the case with acquisition and modelling problems. Figure 1 shows possible ways in which solutions to some problems will benefit or enhance the solution to other problems.

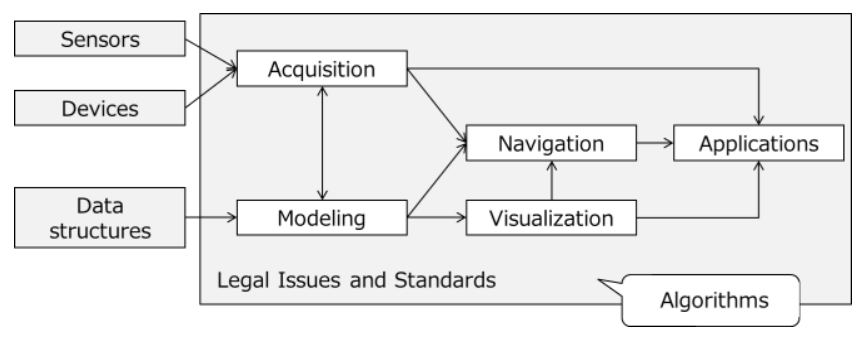

Figure 2: Solving problems in one field of IMM benefits other fields of IMM.

That the solutions to some problems benefit the solution of other problems is rather obvious. However, it is presented here as a reminder that IMM problems do not exist in exclusion to each other, and that solutions have to be developed with the final goal in mind.

A notable departure from this point is problems associated with legal issues and standards. These problems arise as a consequence of IMM. For example acquisition methods or applications that result in a database of imagery give rise to privacy issues. Resolving the privacy issue only allows an acquisition method or application to be used, but it doesn't enhance the method or application.

\section{CONCLUSION}

This paper has considered existing and emerging problems in indoor mapping. The problems are not presented in any order of priority, partly because many of the problems are interlinked.

The purpose of the paper is to set up a framework for discussing problems in indoor mapping and modelling. It is hoped that this framework can then be used as platform for describing indoor mapping and modelling research and developing benchmarks to test solutions for the problems posed here and later.

The problems posed are by no means exhaustive, but they provide an adequate starting point for discussing problems in indoor modelling.

With regard to the problem framework, in near future the ISPRS WG IV/7 intends to do the following:

1. Develop a problem matrix, detailing the problems. This matrix will be the basis of discussion at the Indoor3D conference in December 2013.

2. Develop Empirical Benchmarks for testing solutions to posed problems. Proposed solutions will be evaluated and present best practices will be published. 
3. Yearly evaluation of the problem matrix. Publication of the problem matrix and results from the benchmark tests.

\section{REFERENCES}

Afyouni, I., C. Ray, and C. Claramunt. 2012. Spatial models for context-aware indoor navigation systems: A survey. JOURNAL OF SPATIAL INFORMATION SCIENCE, 4:85-123.

Becker, T., C. Nagel and T. Kolbe. 2009. A Multilayered Space-Event Model for Navigation in Indoor Spaces. 3D GeoInformation Sciences. J. Lee and S. Zlatanova, Springer Berlin Heidelberg: 61-77.

Girard, G., S. Côté, S. Zlatanova, Y. Barette, J. St-Pierre and P. van Oosterom, 2011, Indoor Pedestrian Navigation Using FootMounted IMU and Portable Ultrasound Range Sensors, In: Sensors 2011, Volume 11, pp. 7606-7624

Isikdag, U. 2006, Towards the Implementation of Building Information Models in Geospatial Context, Salford: University of Salford.

Kolodziej, K. W., J. Hjelm, 2006, Local Positioning Systems: LBS Applications and Services, CRC Press, 488 p.

Lee, J., K. Li., S. Zlatanova,, T. Becker, C. Nagel, T. Kolbe, J. Morley 2013. IndoorGML- OGC Candidate Standard for Indoor Navigation; http://www.opengeospatial.org/projects/groups/indoorgmlswg (accessed: 2013 Nov 20.)

NBIMS-1.0. 2007. National building information modeling standard ${ }^{\mathrm{TM}}$. URL: http://www.wbdg.org/pdfs/NBIMSv1_p1.pdf. Accessed: 2013 Nov 19.

Peters, D.; Wu, Y.; Winter, S. (2010): Testing Landmark Selection Theories in Virtual Environment. In: Hölscher, C. et al. (Eds.), Spatial Cognition VII. Lecture Notes in Artificial Intelligence. Springer, Berlin, pp. 54-69.

Smith, J., 1987a. Close range photogrammetry for analyzing distressed trees. Photogrammetria, 42(1), pp. 47-56.

Worboys, M.F., 2011, Modeling indoor space (keynote). Third ACM SIGSPATIAL International Workshop on Indoor Spatial Awareness (ISA 2011), November, Chicago, IL. 2011. http://www.worboys.org/publications/sigspatial\%202011.pdf (accessed:2013, Nov 20) 